\title{
Co-ocurrencia de violencia en el noviazgo en una muestra de jóvenes mexicanos procedentes de zona rural
}

\section{Co-occurrence of dating violence in a sample of Mexicans young people from rural areas}

\author{
Fermina Espinobarros-Nava, Norma Nelida Muñoz-Ponce \\ Universidad Autónoma de Guerrero \\ José Luis Rojas-Solís \\ Facultad de Psicología. Benemérita Universidad Autónoma de Puebla
}

(Rec: marzo de 2018 - Acept: octubre de 2018)

Resumen

El estudio de la violencia en parejas jóvenes ha llamado la atención de la comunidad científica en décadas recientes, no obstante, existen nuevos tipos de violencia, así como poblaciones que no son frecuentemente consideradas en las investigaciones. Por ello el objetivo de esta investigación es analizar la prevalencia y co-ocurrencia de violencia off-line (cara a cara) como on-line (a través de medios electrónicos) en relaciones de pareja de jóvenes procedentes de zona rural. Se realizó un estudio desde un enfoque cuantitativo, con un diseño no experimental, transversal y ex post facto. La muestra estuvo constituida por 231 estudiantes pertenecientes a una universidad pública de Guerrero (México) y provenientes de zona rural, de los cuales 86 fueron hombres y 145 mujeres, con edades comprendidas entre los 18 y 27 años; quienes contestaron las escalas Violence in Adolescents Dating Relationships Inventory (VADRI) y el Cyber Dating Abuse Questionnaire (CDAQ). Entre los resultados destaca la existencia de violencia de distintos tipos en hombres y mujeres, una mayor frecuencia en hombres tanto de violencia sufrida como cometida, así como la co-existencia de diferentes tipos de violencia tanto off-line como on-line en ambos sexos.

Palabras clave: Co-ocurrencia, violencia en el noviazgo, universitario, mexicano, zona rural

\begin{abstract}
In recent decades, the study of violence in young couples has caught the attention of the scientific community. However there are new types of violence as well as population that are not frequently considered in research. Therefore, the objective of this research is to analyze the prevalence and co-occurrence of face-to-face violence (off-line violence) and violence through electronic means (on-line violence) in relationships between young people coming from rural areas. A quantitative study was conducted, with a non-experimental, transversal and ex post facto design. The sample consisted of 231 students from rural areas belonging to a public university in Guerrero (Mexico); 86 men and 145 women, aged between 18 and 27 years, who answered the scales Violence in Adolescents Dating Relationships Inventory (VADRI) and the Cyber Dating Abuse Questionnaire (CDAQ). The results point out the existence of violence of different types in men and women, a greater frequency in men of both violence suffered and committed, as well as the co-ocurrence of different types of off-line and on-line in both sexes.
\end{abstract}

Keywords: Co-ocurrence, Dating violence, College student, Mexican, Rural zone 


\section{Introducción}

En décadas recientes se comenzó a prestar atención a la violencia en las relaciones de pareja de adolescentes y jóvenes como una problemática distinta y separada de lo que se conoce como violencia marital (Castro \& Casique, 2010). Así, se ha identificado que la violencia se puede manifestar en parejas muy jóvenes (González \& Santana, 2001) y que puede presentar una tendencia creciente a medida que se forman relaciones más largas en la edad adulta (Lewis \& Fremouw, 2001). En otras palabras, se sugiere que la violencia en el noviazgo comienza de manera gradual y progresiva por lo que cuanto más antigua es una relación de noviazgo es más el riesgo de sufrir violencia (Rubio-Garay, Carrasco, Amor \& López-González, 2015). De este modo, sería erróneo pensar que la violencia es normal en esta etapa y que desaparecerá con el matrimonio (Valdivia \& González, 2014), tratándose entonces de un fenómeno de alto riesgo para esta población (Jenning et al., 2017; Muñoz-Rivas, González-Lozano, Fernández-González \& Fernández, 2015; Valdivia \& González, 2014). Una vez reconocida la importancia de la violencia en pareja como un problema social y de salud, se puede decir que se ha convertido en un foco de interés (Muñoz-Rivas, et al., 2015).

Pese a la gravedad de lo expuesto, existe un amplio abanico de conceptos que dificultan el establecimiento de una definición compartida sobre la violencia en las relaciones de pareja y, más aún, en las relaciones de noviazgo. En este contexto, en el presente estudio se adoptará la definición empleada por Castro y Casique (2010): "todo acto, omisión, actitud o expresión que genere, o tenga el potencial de generar daño emocional, físico o sexual a la pareja afectiva con la que se comparte una relación íntima sin convivencia ni vínculo marital" (p. 22).

Ahora bien, la comunidad científica se ha preocupado por identificar diversos factores de riesgo que podrían asociarse con una mayor probabilidad de implicarse en relaciones de noviazgo violentas, describiendo variables que ejercen un papel precipitante (provocando o acelerando un episodio violento), facilitador (incrementando la probabilidad de cometer o sufrir agresiones), mediador/modulador (moderando la relación entre una o más variables con la violencia) o inhibidor/ protector (disminuyendo la probabilidad de agredir o protegiendo contra las agresiones (Rubio-Garay et al., 2015).

En cuanto a la tipificación y prevalencia de la violencia, en una revisión sistemática reciente se señala que los tipos de violencia más comunes son la psicológica, física y sexual (Rubio-Garay, López-González, Carrasco \& Amor, 2017). Por una parte, la violencia psicológica es aquella que se encamina a dañar emocionalmente a la pareja y está especialmente dirigida a la autoestima y al autoconcepto. Es considerada una de las violencias más frecuentes, aunque se encuentra invisibilizada (Galán \& Figueroa, 2017) ya que no llega a los golpes y por lo tanto a los jóvenes les puede parecer una conducta "normal" (Moral \& López, 2013). En este tipo de violencia se encuentran tanto mujeres como hombres en los roles de víctima y agresor (Rubio-Garay et al., 2017).

Por otro lado, la violencia física es cualquier acto que la pareja comete de tipo no accidental, provocando o teniendo la intención de causar daño físico a su pareja, observándose lesiones que van de leves a graves. Es el tipo de violencia más fácil de detectar, y se expresa mediante conductas como pellizcar, golpear, empujar y, en casos más graves, ahorcar o intentos de estrangulamiento (Alegría \& Rodríguez, 2015). Es necesario recalcar que este tipo de violencia es la más investigada en el campo de la salud por sus efectos innegables (Galán \& Figueroa, 2017).

La violencia sexual, finalmente, es el uso de medidas de intimidación o coacción contra la pareja, con el fin de mantener relaciones sexuales en contra de su voluntad (Muñoz-Rivas, et al., 2015). Su prevalencia suele ser menor que la psicológica o la física, pero no por ello desdeñable pues, por ejemplo, en un estudio realizado por Ortega, Ortega y Sánchez (2008) se halló que 47.9 \% habían ejercido violencia sexual hacia sus parejas al menos una vez, y el $51.7 \%$ expuso haber sufrido violencia sexual.

En México la violencia en el noviazgo es un fenómeno que se ha estudiado frecuentemente en poblaciones universitarias (Celis-Sauce \& Rojas-Solís, 2015) lo que ha sugerido que se trata de un problema grave pues el $76 \%$ de los jóvenes mexicanos ha sufrido alguna vez violencia psicológica; $15 \%$, violencia física y $16.5 \%$ violencia sexual (IMJ, 2007, citado por Rojas-Solís, 2013).

Por otra parte, el avance de las nuevas tecnologías está generando formas inéditas de convivencia (Serrano-Barquín, \& Ruiz-Serrano, 2013) lo que ha llamado mucho la atención principalmente a los jóvenes, para quienes son herramientas de fácil acceso. Pese a ello, el mal uso de ellas ha favorecido conductas violentas en las relaciones, surgiendo así una nueva forma de violencia llamada ciber-violencia de pareja (García-Sánchez, Guevara-Martínez, Rojas-Solís, Peña-Cárdenas, \& González, 2017). Se trataría de una extensión de la violencia cotidiana a la que están expuestos los y las jóvenes, ya sea como víctima, victimizador o co-agresor, donde las experiencias violentas adquieren nuevas formas de opresión, expresión y discriminación a través de medios electrónicos (Serrano-Barquín \& Ruiz-serrano, 2013). En ese sentido, Gámez-Guadix, Borrajo y Calvete (2018) señalan que esta forma de violencia incluye "control a través de las redes sociales, el robo o el uso indebido de contraseñas, la difusión de secretos o informaciones comprometidas, las amenazas y los insultos públicos o privados a través de las TICs" (p. 2). Es pertinente además, subrayar que el estudio de la violencia on-line poco a poco ha ido llamando la atención en México, al menos así lo señalan los estudios primordialmente con adolescentes, que indican su presencia y la necesidad de su estudio (Jaen-Cortés, Rivera-Aragón, Reidl-Martínez \& García-Méndez, 2017; Lucio-López \& Prieto-Quezada, 2011; Sánchez, Muñoz-Fernández, Lucio \& Ortega-Ruíz, 2017), aunque ciertamente aún son escasas las investigaciones en el país con respecto a esta problemática con universitarios.

Añadido a lo anterior, es conveniente señalar el aspecto de la unidireccionalidad y bidireccionalidad de la violencia, pues hasta hace poco se consideraba a la mujer como única víctima y al hombre como agresor, en la violencia de pareja. No obstante, en una revisión sistemática, Rubio-Garay et al. (2017) hallaron que los hombres no eran los únicos que ejercían violencia contra su pareja, ya que en las mujeres había más prevalencia de la perpetración de violencia psicológica (en los hombres era de tipo sexual), además de que se pudo encontrar que varios de los trabajos revisados detectaron violencia de tipo bidireccional. Pese a esto, varios estudios 
incurren en un sesgo al definir a la mujer como víctima y al varón como victimario, sin embargo, los porcentajes de victimización parecen ser semejantes tanto en hombres como en mujeres (Moral \& Ramos, 2016). En ese orden de ideas, recientemente en otros países estas investigaciones han ido tomando otra dirección, por lo cual se ha empezado a dejar de ver al hombre como el único agresor o perpetrador de violencia en la relación. Al respecto es preciso enfatizar que con esto no se minimizan los índices de violencia sufrida reportados por mujeres, más bien se abren nuevos panoramas de investigación para que con ello se puedan identificar nuevos factores relacionados con la perpetración de violencia y victimización en las relaciones de pareja entre los jóvenes y con ello se puedan proponer programas de prevención e intervención más inclusivos, reales y eficaces.

Mención aparte merece el hecho de que, por cuestiones académicas, la violencia se estudia de manera fraccionada o separada, aunque se ha encontrado que estos diferentes tipos de violencia (en sus distintas modalidades) no suelen presentarse por separado, coexistiendo, lo que se conoce como co-ocurrencia de violencia, es decir la presencia simultánea de diferentes formas de violencia (Hamby \& Grych, 2013).

Respecto del fenómeno de la violencia en el noviazgo, en la actualidad la mayoría de las investigaciones se han centrado en población urbana, razón por la cual este estudio se ha enfocado en jóvenes procedentes de zona rural. En consecuencia, la pregunta que ha guiado la presente investigación es ¿cuál es la prevalencia de los distintos tipos de violencia en el noviazgo de jóvenes universitarios guerrerenses procedentes de zona rural y la relación entre ellos? Para contestar esta cuestión se han trazado tres objetivos: 1) Conocer la prevalencia de violencia cometida o sufrida, tanto off-line como on-line, en las relaciones de pareja de los y las participantes. 2) Determinar qué sexo comete con mayor frecuencia violencia off-line y on-line en sus relaciones de noviazgo. 3) Analizar la relación que existe entre los diferentes tipos de violencia off-line y on-line. Con el fin de alcanzar estos objetivos, se han elaborado las siguientes hipótesis 1.1 Existirá violencia en las relaciones de parejas de estudiantes, 2.1 Los hombres cometerán con mayor frecuencia violencia hacia sus parejas, 2.2 Las mujeres señalarán sufrir con mayor frecuencia violencia que los hombres. 3.1 Existirán correlaciones significativas y positivas entre los diferentes tipos de violencia.

\section{Método}

\section{Participantes}

Se llevó a cabo una investigación con un enfoque cuantitativo, utilizando un diseño de investigación no experimental, transversal y ex post facto, con alcances exploratorios, descriptivos y correlacionales. La muestra se seleccionó intencionalmente y fue constituida por 231 estudiantes pertenecientes a la Universidad Autónoma de Guerrero provenientes de una zona rural, de los cuales $37.2 \%$ fueron hombres $(n=86)$ y $62.8 \%$ mujeres $(n=145)$, entre los 18 y 27 años de edad $(M=21,72 ; D T=$ $1,796)$ y de diversas carreras de la misma universidad. El criterio de inclusión fue tener o haber tenido una relación de pareja que haya durado al menos un mes de duración. Es preciso señalar que, para el presente trabajo, de la muestra total se descartaron las respuestas de personas que señalaron otro tipo de relación que no fuera de noviazgo, personas no heterosexuales y que no pertenecieran a una zona rural.

\section{Instrumentos}

En el presente estudio se destinó un apartado inicial de la encuesta para solicitar información sociodemográfica de los participantes como la edad, sexo, institución de procedencia, carrera, grado escolar, zona (rural o urbana), tipo de relación de pareja, sexo de su pareja, nivel educativo del padre y nivel educativo de la madre, entre otros.

Posteriormente, se aplicó el inventario llamado Violence in Adolescents Dating Relationships Inventory (VADRI [versión adaptada] Aizpitarte, Alonso-Arbiol, Van de Vijver, Perdomo, Gálvez-Sobral \& García-López, 2017), el cual fue validado en población mexicana y permite evaluar la violencia en el noviazgo a través 19 ítems de doble naturaleza (victimización y perpetración) con un rango de respuesta que va de 1 al 10, donde 1=Nunca hasta $10=$ Siempre, misma que se divide en tres factores: 1) violencia directa/severa (6 ítems) que representa las conductas directas y severas de la violencia en el noviazgo; 2) conductas de control/aislamiento (8 ítems) el cual está conformado por aquellos comportamientos de control e intentos de aislamiento hacia la pareja, incluyendo aquellos realizados con el uso de las nuevas tecnologías; y 3) violencia indirecta/verbal (5 ítems) que refiere a varios comportamientos violentos psicológicos relacionados con actos verbales de desacreditar a la pareja, ya sea en presencia, o no, de esta.

Así mismo, se utilizó el Cyber Dating Abuse Questionnaire (CDAQ, Borrajo, Gámez-Guadix, Pereda \& Calvete, 2015), el cual detecta un amplio rango de comportamientos de ciber-violencia en el noviazgo, tales como amenazas, robo de identidad, control y humillación. Esta escala se compone por cuarenta ítems de doble naturaleza en los cuales veinte reactivos evalúan la frecuencia de perpetración; y los otros veinte, victimización, a través de una escala tipo Likert con un recorrido de 6 puntos, donde 1=Nunca, $2=$ No en el último año, pero si anteriormente, $3=$ Rara vez, $4=A$ veces, $5=$ Con frecuencia y $6=$ Casi siempre. A su vez, la escala está dividida en dos sub-escalas: control de la pareja, cometido y sufrido, así como agresión directa, cometido y sufrido. La fiabilidad obtenida en el VADRI y CDAQ, tanto para la muestra total como la muestra de hombres y mujeres, puede observarse en la Tabla 1.

\section{Procedimiento}

Se contactó a los participantes de manera incidental e individual, solicitando su colaboración para contestar un cuestionario en línea. Este fue realizado en el programa Google forms, conteniendo la presentación del estudio (naturaleza, objetivos y criterios de inclusión) así como el consentimiento informado donde se recalcaba el carácter voluntario de la participación, el anonimato y la confidencialidad de la información obtenida. Los datos recolectados se almacenaron y analizaron en el programa SPSS (Statistical Package for the Social Sciences) versión 21.

\section{Análisis estadístico}

Inicialmente, se calcularon todos los índices de consistencia interna de los instrumentos utilizados mediante el Alpha de Cronbach, posteriormente se obtuvieron los descriptivos de las principales variables sociodemográficas y de los instrumentos implementados, así como las frecuencias de las respuestas por ítems. Acto seguido se realizó la prueba de normalidad de las respuestas por medio del índice de Kolmogorov-Smirnov obteniéndose no normalidad en los datos, razón por la que se utilizaron análisis no paramétricos para detectar diferencias 
entre sexos, por medio de la prueba $U$ de Mann-Whitney, y el establecimiento de la asociación entre las variables, por medio del coeficiente de correlación de Spearman (rho).

\section{Resultados}

En primer término, se presentan los datos estadísticos descriptivos e índices de confiabilidad (Tabla 1) de las escalas/subescalas y las principales variables sociodemográficas de la muestra total $(N=231)$, donde sobresale la obtención de índices altos en la mayoría de las subescalas, a excepción de la variable perteneciente a la violencia sufrida y cometida indirecta verbal, tanto en hombres como en mujeres, y en la variable relacionada con la violencia cometida directa severa, submuestra de mujeres.
En cuanto a la presencia (FP) y ausencia (FA) de violencia en hombres y mujeres es importante mencionar que en todas las variables se encontró la presencia de violencia al menos una vez, donde se destaca el tipo de violencia relacionado con el control cometido $(n=106)$ y el control sufrido $(n=100)$ con alta presencia en mujeres; en cuanto a los hombres, la violencia relacionada con el control sufrido fue el que más prevaleció $(n=75)$ así como la violencia indirecta sufrida verbal $(n=70)$.

Por otra parte, los datos obtenidos muestran mayor ausencia de violencia en la violencia cometida directa severa en mujeres $(n=90)$ y en hombres $(n=29)$, del mismo modo en la violencia sufrida directa en mujeres $(n=82)$ y hombres $(n=28)$.

Tabla 1

Índices de confiabilidad y descriptivos

\begin{tabular}{|c|c|c|c|c|c|c|}
\hline & $\alpha$ & $M$ & $M d$ & $D T$ & $F A$ & $F P$ \\
\hline Edad & - & 21.72 & 22 & 1.79 & - & - \\
\hline Mujeres & - & 21.67 & 22 & 1.59 & - & - \\
\hline Hombres & - & 21.81 & 22 & 2.10 & - & - \\
\hline Violencia Sufrida Directa Severa & .91 & 1.73 & 1.17 & 1.30 & - & - \\
\hline Mujeres & .92 & 1.55 & 1.00 & 1.17 & 82 & 63 \\
\hline Hombres & .90 & 2.02 & 1.58 & 1.47 & 28 & 58 \\
\hline Violencia Cometida Directa Severa & .90 & 1,56 & 1,00 & .95 & - & - \\
\hline Mujeres & .87 & 1.39 & 1.00 & .83 & 90 & 55 \\
\hline Hombres & .92 & 1.84 & 1.42 & 1.08 & 29 & 57 \\
\hline Violencia Sufrida Control/Aislamiento & .97 & 3.23 & 2.38 & 2.45 & - & - \\
\hline Mujeres & .96 & 2.63 & 1.50 & 2.20 & 46 & 99 \\
\hline Hombres & .96 & 4.13 & 3.88 & 2.58 & 14 & 72 \\
\hline Violencia Cometida Control/Aislamiento & .97 & 3.01 & 1.88 & 2.29 & - & - \\
\hline Mujeres & .97 & 2.61 & 1.38 & 2.15 & 56 & 89 \\
\hline Hombres & .96 & 3.68 & 3.19 & 2.38 & 19 & 67 \\
\hline Violencia Sufrida Indirecta Verbal & .86 & 2.28 & 1.60 & 1.54 & - & - \\
\hline Mujeres & .88 & 1.99 & 1.40 & 1.39 & 54 & 91 \\
\hline Hombres & .84 & 2.79 & 2.50 & 1.65 & 16 & 70 \\
\hline Violencia Cometida Indirecta Verbal & .84 & 2.02 & 1.40 & 1.26 & - & - \\
\hline Mujeres & .79 & 1.86 & 1.40 & 1.16 & 59 & 86 \\
\hline Hombres & .89 & 2.30 & 1.70 & 1.38 & 22 & 64 \\
\hline Control Sufrido & .95 & 2.40 & 2.11 & 1.33 & - & - \\
\hline Mujeres & .95 & 2.16 & 1.67 & 1.25 & 45 & 100 \\
\hline Hombres & .94 & 2.82 & 2.89 & 1.36 & 11 & 75 \\
\hline Control Cometido & .95 & 3.39 & 2.00 & 1.37 & - & - \\
\hline Mujeres & .93 & 2.13 & 1.67 & 1.18 & 39 & 106 \\
\hline Hombres & .96 & 2.82 & 2.44 & 1.55 & 18 & 68 \\
\hline Agresión Directa Sufrida & .94 & 1.47 & 1.09 & .76 & - & - \\
\hline Mujeres & .94 & 1.32 & 1.00 & .64 & 77 & 68 \\
\hline Hombres & .93 & 1.72 & 1.41 & .88 & 25 & 61 \\
\hline Agresión Directa Cometida & .94 & 1.43 & 1.09 & .75 & - & - \\
\hline Mujeres & .93 & 1.29 & 1.00 & .62 & 78 & 67 \\
\hline Hombres & .95 & 1.66 & 1.27 & .90 & 27 & 59 \\
\hline
\end{tabular}

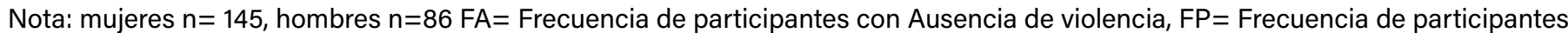
con Presencia de violencia (al menos una vez) 
Diferencias entre mujeres y hombres en la perpetración y victimización de violencia

En segundo lugar, en la Tabla 2 se muestra que en todas las variables evaluadas se hallaron diferencias significativas entre

Tabla 2

Diferencias por sexo en las variables estudiadas

\begin{tabular}{|c|c|c|c|c|c|c|c|c|c|c|}
\hline & & \multicolumn{2}{|c|}{$\begin{array}{l}\text { Mujeres } \\
(n=145)\end{array}$} & \multicolumn{2}{|c|}{$\begin{array}{c}\text { Hombres } \\
(n=86)\end{array}$} & \multirow[b]{2}{*}{$p$} & \multirow[b]{2}{*}{$U$} & \multirow[b]{2}{*}{$Z$} & \multirow[b]{2}{*}{$r$} & \multirow[b]{2}{*}{ Psest } \\
\hline & & $M d$ & Rango & $M d$ & Rango & & & & & \\
\hline \multicolumn{11}{|c|}{ Violencia Directa Severa (VADRI) } \\
\hline Sufrida & & 1.00 & 103.79 & 1.58 & 136.59 & .000 & 4464.00 & -3.821 & -0.25 & 0.35 \\
\hline Cometida & & 1.00 & 101.44 & 1.42 & 140.55 & .000 & 4124.00 & .4 .630 & -0.30 & 0.33 \\
\hline \multicolumn{11}{|c|}{ Violencia Control Aislamiento (VADRI) } \\
\hline Sufrida & & 1.50 & 101.73 & 3.88 & 140.05 & .000 & 4166.50 & -4.251 & -0.27 & 0.33 \\
\hline Cometida & & 1.38 & 104.13 & 3.19 & 136.01 & .000 & 4514.50 & -3.566 & -0.23 & 0.36 \\
\hline \multicolumn{11}{|c|}{ Violencia Indirecta Verbal (VADRI) } \\
\hline Sufrida & & 1.40 & 102.01 & 2.50 & 139.59 & .000 & 4206.50 & -4.194 & -0.27 & 0.33 \\
\hline Cometida & & 1.40 & 107.07 & 2.89 & 131.06 & .007 & 4939.50 & -2.700 & -0.17 & 0.39 \\
\hline \multicolumn{11}{|c|}{ Control (CDAQ) } \\
\hline Sufrido & & 1.67 & 103.21 & 2.89 & 137.56 & .000 & 4380.50 & -3.805 & -0.25 & 0.35 \\
\hline Cometido & & 1.67 & 105.59 & 2.44 & 133.56 & .002 & 4725.00 & -3.100 & -0.20 & 0.37 \\
\hline $\begin{array}{l}\text { Agresión } \\
\text { (CDAQ) }\end{array}$ & Directa & & & & & & & & & \\
\hline Sufrida & & 1.00 & 101.44 & 1.41 & 140.55 & .000 & 4124.00 & -4.500 & -0.29 & 0.33 \\
\hline Cometida & & 1.00 & 102.94 & 1.27 & 138.02 & .000 & 4341.00 & -4.055 & -0.26 & 0.34 \\
\hline
\end{tabular}

\section{Correlaciones entre los diversos tipos de violencia}

Por otro lado, en la Tabla 3 se muestran los resultados obtenidos por medio del índice de correlación de Spearman, para ello se separa los resultados por sexo y se enfatizan los hallazgos principales en la violencia 1) off-line, 2) on-line y 3) off-line con on-line.

En el caso de las mujeres, en la violencia off-line, se encontró una fuerte asociación positiva entre violencia sufrida y cometida en relación a la violencia sobre control y aislamiento ( $r$ ho = $\left..827^{* *}, n=145, p<.01\right)$; del mismo modo, se encontró una fuerte asociación significativa entre la perpetración de control/aislamiento y violencia indirecta verbal cometida ( $r h o=.806^{* *}$, $n=145, p<.01)$. Por otra parte, con relación a la violencia on-line se pudo encontrar que el control cometido y el sufrido tienen una fuerte significancia y grado de asociación (rho $=.862^{* *}$, $n=145, p<.01)$, así como también, la agresión directa sufrida con la agresión directa cometida ( $\left.r h o=.800^{* *}, \mathrm{n}=145, p<.01\right)$. No obstante lo anterior, también se pudo encontrar asociaciones significativas entre la violencia off-line y la on-line por lo que el tipo de violencia relacionada con el control y aislamiento sufrido obtuvo una correlación positiva y significativa con el control on-line sufrido ( $r h o=.856^{* *}, n=145, p<.01$ ), mientras que la violencia cometida en relación al control y aislamiento se relacionó significativamente con el control on-line cometido (rho $\left.=.851^{* *}, n=145, p<, 01\right)$. los sexos. Así, los hombres señalaron cometer mayormente estos tipos de violencia, sin embargo, también indicaron sufrirla, es decir reconocieron ejercer y sufrir con mayor frecuencia distintos tipos de violencia tanto off-line como on-line.

.


Tabla 3

Correlaciones entre violencia off-line $y$ on-line

\begin{tabular}{|c|c|c|c|c|c|c|c|c|c|c|}
\hline & 1 & 2 & 3 & 4 & 5 & 6 & 7 & 8 & 9 & 10 \\
\hline 1 & - & $.640^{* *}$ & $.700 * *$ & $.617^{* *}$ & $.778^{* *}$ & $.724^{* *}$ & $.638^{* *}$ & $.611^{* *}$ & $.697^{* *}$ & $.562^{* *}$ \\
\hline 2 & $.648^{* *}$ & - & $.636^{* *}$ & $.624^{* *}$ & $.605^{* *}$ & $.644^{* *}$ & $.556^{* *}$ & $.556^{* *}$ & $.574 * *$ & $.554^{* *}$ \\
\hline 3 & $.647^{* *}$ & $.484^{* *}$ & - & $.827^{* *}$ & $.780^{* *}$ & $.745^{* *}$ & $.856^{* *}$ & $.808^{* *}$ & $.697^{* *}$ & $.646^{* *}$ \\
\hline 4 & $.447^{* *}$ & $.692^{* *}$ & $.773^{* *}$ & - & $.662^{* *}$ & $.806^{* *}$ & $.744^{* *}$ & $.851^{* *}$ & $.626 * *$ & $.664^{* *}$ \\
\hline 5 & $.689^{* *}$ & $.630 * *$ & $.765^{* *}$ & $.705^{* *}$ & - & $.754^{* *}$ & $.714^{* *}$ & $.653^{* *}$ & $.730 * *$ & $.643^{* *}$ \\
\hline 6 & $.551^{* *}$ & $.832^{* *}$ & $.533^{* *}$ & $.772^{* *}$ & $.691^{* *}$ & - & $.687^{* *}$ & $.738^{* *}$ & $.621^{* *}$ & $.668^{* *}$ \\
\hline 7 & $.390^{* *}$ & $.525^{* *}$ & $.761^{* *}$ & $.756^{* *}$ & $.584^{* *}$ & $.507^{* *}$ & - & $.862^{* *}$ & $.758^{* *}$ & $.711^{* *}$ \\
\hline 8 & $.331^{* *}$ & $.615^{* *}$ & $.681^{* *}$ & $.827^{* *}$ & $.626^{* *}$ & $.599 * *$ & $.892^{* *}$ & - & $.676^{* *}$ & $.702^{* *}$ \\
\hline 9 & $.390^{* *}$ & $.581^{* *}$ & $.527^{* *}$ & $.593^{* *}$ & $.577^{* *}$ & $.546 * *$ & $.739 * *$ & $.747^{* *}$ & - & $.800^{* *}$ \\
\hline 10 & $.363^{* *}$ & $.599 * *$ & $.486 * *$ & $.564^{* *}$ & $.557^{* *}$ & $.559 * *$ & $.656^{* *}$ & $.797^{* *}$ & $.839 * *$ & - \\
\hline
\end{tabular}

Nota: ${ }^{*} \mathrm{p}<.05 ;{ }^{* *} \mathrm{p}<.001$

Los valores correspondientes a las mujeres $(n=145)$ están por encima de la diagonal; los de los hombres $(n=86)$ por debajo.

1=Violencia sufrida directa severa; $2=$ Violencia cometida directa severa; $3=$ Violencia sufrida control/aislar; $4=$ Violencia cometida control/aislar; $5=$ Violencia sufrida directa verbal; $6=$ Violencia cometida indirecta verbal; $7=$ Control sufrido; $8=$ Control cometido; $9=$ Agresión directa sufrida; $10=$ Agresión directa cometida.

\section{Discusión}

El presente estudio se encaminó a analizar la prevalencia y, en su caso, la relación entre distintos tipos de violencia en el noviazgo de jóvenes universitarios guerrerenses procedentes de zona rural. Al respecto, es preciso señalar que a la luz de los resultados obtenidos sí se halló existencia de distintos tipos de violencia, co-ocurrencia de los mismos, y que tanto hombres como mujeres han sufrido y cometido violencia en sus relaciones de pareja.

Aunado a esto, se encontró que la violencia que más prevaleció en los hombres fue la violencia indirecta sufrida verbal y el control sufrido, mientras que en las mujeres fue el control cometido y el sufrido, lo cual tiene correspondencia con lo encontrado por Rubio-Garay et al. (2017) quienes hallaron que las mujeres tienden a ejercer mayormente violencia psicológica hacia sus parejas. Sin embargo, difiere de lo hallado por Pazos, Oliva y Hernando (2014) donde la violencia psicológica fue utilizada por ambos sexos, sin olvidar que fueron las participantes quienes ejercieron con más frecuencia violencia física; y los hombres, violencia sexual y relacional.

Se esperaba encontrar que los hombres cometerían con mayor frecuencia violencia hacia sus parejas, lo cual se pudo comprobar ya que los resultados indicaron que fueron los hombres quienes reconocieron ejercerla con mayor frecuencia, aunque también fueron ellos quienes reportaron sufrir más este tipo de conductas. En ese mismo sentido, algunos estudios hacen énfasis en que los hombres son quienes cometen con mayor frecuencia violencia, no obstante, también se ha evidenciado que los porcentajes de victimización y perpetración son semejantes en mujeres y hombres (Moral \& Ramos, 2016). El mayor grado de violencia sufrida y cometida reportado por los hombres en comparación con las mujeres, tal vez se deba a la normalización y aceptación de estos tipos de violencia, llegando a formarse un clima de aceptación y tolerancia a la violencia, debido a la influencia de ciertos valores culturales que contribuirían a la percepción de la violencia como una forma "normal" y válida de resolver los problemas
(Saldívar, Ramos \& Saltijeral, 2004).

En relación con lo anterior, es conveniente señalar que la existencia de conductas asociadas a la vigilancia y control por medios electrónicos no son exclusivas de un sexo o edad, pues investigaciones con adolescentes mexicanos ya lo habían indicado, como también su peligrosidad por su normalización, confundiéndose con interés por la pareja (Jaen-Cortés et al., 2017; Lucio-López \& Prieto-Quezada, 2011).

Por otra parte, también se pretendía confirmar que las mujeres señalarían sufrir con mayor frecuencia violencia en comparación con los hombres, empero se encontró mayor prevalencia de violencia sufrida en los hombres, lo cual sugiere una posible bidireccionalidad en este tipo de poblaciones. Al respecto es preciso señalar que, por un lado, esa situación no implica que ambos sexos sufran las mismas consecuencias de la violencia y, por otro, que debido a la reticencia para aceptar el carácter bidireccional de la violencia en las relaciones de pareja, aún son escasas las investigaciones que plantean la posibilidad de ir más allá de la dicotomía hombre-agresor y mujer-víctima, a pesar de que en otras partes del mundo ya existan estudios sobre la violencia cometida y sufrida por parte de hombres y mujeres desde hace varios años (Rubio-Garay, López-González, Saúl \& Sánchez-Elvira-Paniagua, 2012)

Así mismo, se esperaba confirmar la existencia de asociaciones significativas y positivas entre los diferentes tipos de violencia, algo que pudo comprobarse. De estos datos es preciso señalar la co-ocurrencia de violencia on-line y off-line, lo cual encuentra similitud con investigaciones previas, como la realizada por Yahner, Dank, Zweig y Lachman (2014), quienes hallaron la concurrencia de varios tipos de violencia off-line y on-line; o la pesquisa realizada por Gámez-Guadix, Borrajo y Calvete (2018) que apuntó la co-existencia de violencia on-line con la violencia off-line de tipo psicológica y física.

En otro orden de ideas, es importante mencionar algunas de las limitaciones del estudio, tales como su enfoque cuantitativo, que no permitió ahondar más allá de lo que se pregunta en 
las escalas, así como la posible deseabilidad de las respuestas. Por otra parte, el diseño trasversal impide saber cómo va evolucionando este tipo de relaciones a lo largo del tiempo. Añadido a lo anterior, es preciso reconocer que el tamaño, selección, composición y representatividad de la muestra no permite la generalización de los resultados.

En cuanto a las futuras líneas de investigación, se podrían indicar algunas sugerencias como la ampliación y diversificación de la muestra, de tal manera que además de agregar la característica de procedencia de zona rural, se puedan agregar aspectos diversos como jóvenes adolescentes, personas homosexuales, jóvenes que no estén estudiando, por ejemplo.

A modo de cierre y en el entendido de que aún son escasos los estudios sobre violencia en parejas de jóvenes procedentes de zona rural, esta investigación se centró en la exploración del fenómeno corroborando la existencia y co-ocurrencia de violencia off-line y on-line sugiriendo, además, una posible bidireccionalidad de estos comportamientos. Se trata, pues, de un aporte modesto, pero no desdeñable, al fortalecimiento del corpus sobre la materia en el país y la región.

\section{Referencias}

Aizpitarte, A., Alonso-Arbiol, I., Van de Vijver, F. J., Perdomo, M. C., Gálvez-Sobral, J. A., \& García-López, E. (2017). Development of a dating violence assessment tool for late adolescence across three countries: The Violence in Adolescents Dating Relationships Inventory (VADRI). Journal of interpersonal Violence, 30, 1-21. doi:10.1177/088626051593543

Alegría, M., \& Rodríguez, A. (2015). Violencia en el noviazgo: Perpetración, victimización y violencia mutua. Una revisión. Actualidades en psicología, 29(118), 57-72. doi:10.15517/ap.v29i118.16008

Borrajo, E., Gámez-Guadix, M., Pereda, N., \& Calvete, E. (2015). The development and validation of the Cyber Dating Abuse Questionnaire among young couples. Computers in Human Behavior, 48, 358-365. doi:10.1016/j.chb.2015.01.063

Castro, R., \& Casique, I. (2010). Violencia en el noviazgo entre los jóvenes mexicanos. Cuernavaca, México: UNAM.

Celis-Sauce, A., \& Rojas-Solís, J. L. (2015). Adolescentes mexicanos como víctimas y perpetradores de violencia en el noviazgo. ReiDoCrea, 4(9), 60-65.

Galán, J. S., \& Figueroa, M. R. (2017). Gaslighting: La invisible violencia psicológica. UARICHA Revista de Psicología, 14(32), 53-60.

Gámez-Guadix, Borrajo \& Calvete. (2018). Abuso, control y violencia en la pareja a través de internet y los smartphones: características, evaluación y prevención, Papeles del psicólogo (artículo en prensa).

García-Sánchez, P. V., Guevara-Martínez, C., Rojas-Solís, J. L., Peña-Cárdenas., \& González, V. G. (2017). Apego y Ciber-violencia en la pareja de adolescentes. $R e$ vista INFAD de Psicología, 1(1), 541-550.

González, R., \& Santana, J. D. (2001). La violencia en parejas jóvenes. Psicothema, 13(1), 127-131.

Hamby, S., \& Grych, J. (2013). The web of violence. Exploring connections among different forms of interpersonal violence and abuse. Dordrecht (The Netherlands): Springer.

Summa Psicológica UST (CC - BY - 3.0)

ISSN: 0718-0446 / ISSNe: 0719-448x

http://summapsicologica.cl/
Jaen-Cortés, C. I., Rivera-Aragón, S., Reidl-Martínez, L.M., \& García-Méndez, M. (2017). Violencia de pareja a través de medios electrónicos en adolescentes mexicanos. Acta de Investigación Psicológica, 7(1), 2593-2605. doi:10.1016/j.aipprr.2017.01.001

Jennings, W. G., Okeem, C., Piquero, A. R., Sellers, C. S., Theobald, D., \& Farrington, D. P. (2017). Aggression and Violent Behavior, 33, 107-125. doi:10.1016/j. avb.2017.01.007

Lewis. S. F., \& Fremouw, W. (2001). Dating violence: a critical review of the literature. Clinical Psychology Review, 21(1), 105-127.

Lucio-López, L., \& Prieto-Quezada, M. (2011). Violencia en el ciberespacio en las relaciones de noviazgo adolescente. Un estudio exploratorio en estudiantes mexicanos de escuelas preparatorias. Revista de Educación y Desarrollo, 31, 61-72.

Moral, J., \& López, F. (2013). Violencia de pareja en personas que viven o no con su pareja y en ambos sexos. Psicogente, 16(30), 296-310.

Moral, J., \& Ramos, B. S., (2016). Machismo, victimización y perpetración en mujeres y hombres mexicanos. Estudios sobre las culturas contemporáneas, 22(43), 37-66.

Muñoz-Rivas, M., González-Lozano, P., Fernández-González, L. \& Fernández, S. (2015). Violencia en el noviazgo. Realidad y prevención. Madrid: Ediciones Pirámide.

Ortega, R., Ortega, F. J., \& Sánchez, V. (2008). Violencia sexual entre compañeros y violencia en parejas adolescentes. International Journal of Psycology and Psychological therapy, 8(1), 63-72.

Pazos, M., Oliva, A., \& Hernando, A. (2014). Violencia en relaciones de pareja de jóvenes y adolescentes. Revista latinoamericana de psicología, 46(3), 148-159.

Rojas-Solís, J. L. (2013). Violencia en el noviazgo y sociedad mexicana posmoderna. Algunos apuntes sobre la figura del agresor y las agresiones bidireccionales. Uaricha Revista de Psicología, 10(22), 1-19.

Rubio-Garay, F., Carrasco, M. A., Amor, J. P., \& López-González, M. A. (2015). Factores asociados a la violencia en el noviazgo entre adolescentes: Una revisión crítica. Anuario de psicología jurídica, 25(1), 47-56. doi:10.1016/j.apj.2015.01.001

Rubio-Garay, F., López-González, M. A., Carrasco, M. A., \& Amor, P. J. (2017). Prevalencia de la violencia en el noviazgo: una revisión sistemática. Papeles del psicólogo, 38(2), 135-147. doi:10.23923/pap.psicol2017.2831.

Rubio-Garay, F., López-González, M. A., Saúl, L. A. \& Sánchez-Elvira-Paniagua, A. (2012). Direccionalidad y expresión de la violencia en las relaciones de noviazgo de los jóvenes. Acción Psicológica, 9(1), 6170. doi:10.5944/ap.9.1.437

Saldívar, G., Ramos, L., \& Saltijeral, M. T. (2004). Validación de las escalas de aceptación de la violencia y de los mitos de violación en estudiantes universitarios. Salud Mental, 27(6), 40-49.

Sánchez, V., Muñoz-Fernández, N., Lucio, L. A., \& Ortega-Ruíz, R. (2017). Ciberagresión en parejas adolescentes: Un estudio transcultural España-México. Revista Mexicana de Psicología, 34(1), 46-54.

Serrano-Barquín, R., \& Ruiz-Serrano, E. (2013). Violencia simbólica en internet. Raximhai, 9(3), 121-139. 
Valdivia, M. P. \& González, L. A. (2014). Violencia en el noviazgo y pololeo: Una actualización proyectada hacia la adolescencia. Revista de Psicología, 32(2), 330355.

Yahner, J., Dank, M., Zweig. J. M., \& Lachman P. (2014). The co-occurrence of physical and cyber dating violence and bullying among teens. Journal of Interpersonal Violence, 30(7), 1079-89. doi:10.1177/0886260514540324 